\title{
Simulação bidimensional do fluxo da Geleira Lange, Ilha Rei George, Antártica
}

H.H.C. BARBOZA, A.L. de BORTOLI, R.D. da CUNHA, IM-DMPA/PPGMAP, UFRGS, Porto Alegre-RS, Brasil

J.C. SIMÕES, Departamento de Geografia, Instituto de Geociências, UFRGS, Porto Alegre-RS, Brasil.

\begin{abstract}
Resumo. As bacias de drenagem glacial na Ilha Rei George recuaram mais que um quilômetro durante os últimos 40 anos, perdendo em torno de $7 \%$ da cobertura de gelo original. Esta redução ocorreu concomitantemente com o aumento da temperatura atmosférica em torno de $1.1{ }^{\circ} \mathrm{C}$. Neste trabalho, apresenta-se, no contexto de diferenças finitas, um modelo numérico para simulação do fluxo da geleira de descarga Lange (Ilha Rei George, Antártica). Estima-se o estado de equilíbrio e as respostas morfológicas para mudanças na taxa de acumulação de gelo. O modelo computa a distribuição de velocidades ao longo da linha central longitudinal principal usando a lei do fluxo do gelo para calcular a deformação interna sem deslizamento basal e as respostas às mudanças na acumulação líquida. Os resultados sugerem uma geleira próximo ao estado estacionário.
\end{abstract}

\section{Introdução}

As bacias de drenagem glacial na Ilha Rei George recuaram mais de um quilômetro nos últimos 40 anos, perdendo cerca de $7 \%$ da cobertura de gelo original ([11]). Esta perda foi concomitante ao aumento da temperatura de $1.1^{\circ} \mathrm{C}([5])$ atmosférica média regional. As Shetlands do Sul apresentam massas de gelo muito sensíveis às mudanças climáticas, visto que essas se encontram perto do ponto de fusão sob pressão. Apresentando o maior recuo, a Geleira Lange perdeu aproximadamente 2 $\mathrm{km}^{2}$ de sua frente $([2])$.

O fluxo desta geleira foi modelado para estimar e prever o seu estado de equilíbrio e a sua resposta a mudanças na taxa de acumulação. Obtemos resultados para a velocidade e a distribuição da temperatura do gelo através de um modelo bidimensional. Os dados, utilizados no modelo, foram obtidos através do trabalho de campo realizado pelo Laboratório de Pesquisas Antárticas e Glaciológicas no verão de 1999/2000 (Operação Antártica XVIII). 


\section{Glaciologia da Bacia de Drenagem Modelada - Geleira Lange}

A Lange é uma geleira de maré, ou seja, possui seu término no mar, geralmente em uma baía ou em um fiorde, onde ocorre o processo chamado desprendimento (calving), isto é, separação de blocos de gelo dentro d'água formando icebergs. Geralmente, quando há redução no despreendimento, o balanço de massa se torna positivo e, então, inicia-se o avanço da geleira. A Lange também pode ser considerada como uma geleira de descarga; a sua bacia de drenagem tem área total de $28.3 \mathrm{~km}^{2}$.

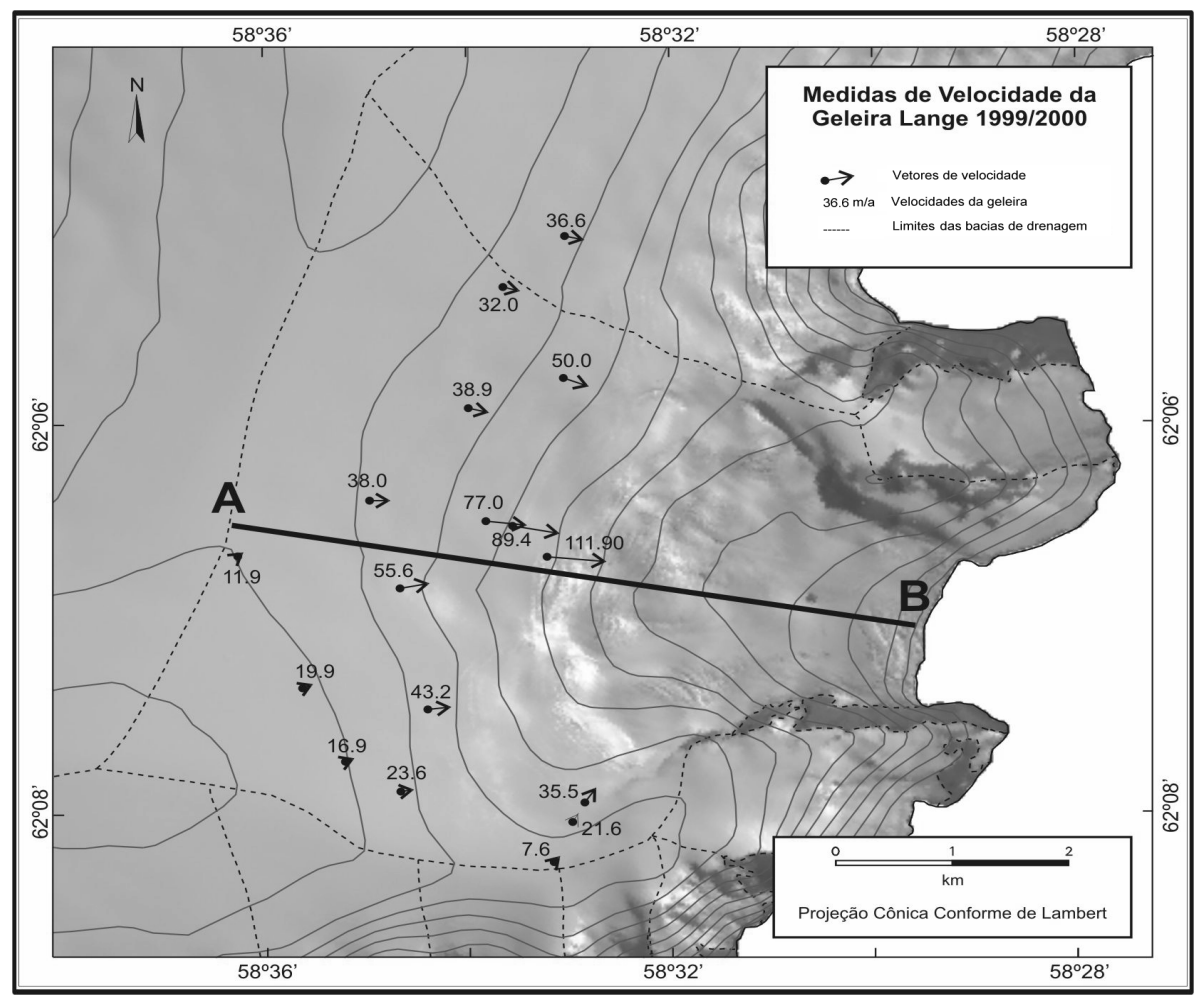

Figura 1: Imagem de satélite mostrando o movimento superficial da Geleira de Descarga Lange com seus respectivos vetores velocidade e o perfil A-B.

O comprimento longitudinal desta geleira, na parte central, é de $9 \mathrm{~km}$ e sua largura, na porção intermediária, de $6,5 \mathrm{~km}$; a sua frente tem $2 \mathrm{~km}$ de largura, desembocando no interior da Baía do Almirantado (costa sul da Ilha Rei George).

A bacia de drenagem foi determinada através de imagem de satélite ([11]), através de pontos de radioecossondagem ([6], [7]) e medidas de velocidade.

A morfologia subglacial foi construída com dados obtidos através da técnica 
de radioecosondagem. Essa técnica é utilizada para levantamentos glaciológicos, gerando informações sobre o perfil do embasamento rochoso de uma área glaciada, da espessura e da estrutura interna do gelo ([8]). No verão de 1999/2000, durante a XVIII Operação Antártica Brasileira, foram implantadas 21 estacas na bacia de drenagem da geleira Lange, com o intuito de determinar a velocidade da superfície do gelo.

O equipamento utilizado foi um GPS diferencial (Sistema Global de Posicionamento Diferencial) com precisão centimétrica. Dois aparelhos GPS (Magellan PRO$M A R K X C M$ ) receptores, um fixo num ponto com coordenadas geográficas conhecidas e outro em movimento ou na posição para medir, foram usados. Os resultados confirmaram os dados teóricos; isto é, estacas próximas aos limites da bacia de drenagem, determinada através de imagens de satélites, movem-se mais lentamente do que as estacas no centro da bacia.

\section{Hipóteses adotadas no modelo}

Para obter o perfil da superfície foram utilizados os dados de altitude e para o subglacial foi realizada a subtração da altitude pela espessura do gelo, obtendo assim ambos perfis. Os dados da topografia superficial foram determinados através do uso das altitudes do perfil A-B ao longo do eixo principal da geleira, conforme Figura 2. A construção subglacial foi feita da mesma forma, porém foram utilizados dados coletados através da radioecossondagem.

Um dos métodos empregados para deformações de sólidos e fluidos é o de diferenças finitas ([1]), que é simples e eficiente. A fim de simular o fluxo do gelo, as equações do movimento na sua forma geral podem ser empregadas. Entretanto, os dados experimentais mostram que as velocidades são suficientemente pequenas (menos de $1 \mathrm{~m}$ por dia) na geleira Lange; isto permite fazer algumas simplificações. Desta maneira, a equação da energia é empregada para obter variações da temperatura; a conservação da massa é baseada na variação transversal da área. A velocidade média de uma coluna vertical de gelo é definida por

$$
u=v_{i}+v_{b},
$$

onde $v_{i}$ é a velocidade da deformação interna e $v_{b}$ a velocidade do deslizamento

basal. É claro que poderemos ter casos particulares, podendo reduzir esta relação a $u=v_{i}$ em grande parte do manto de gelo Antártico; $v_{i}$ pode ser obtido usando a relação empírica $([9])$

$$
v_{i}=A_{1} \tau_{b}^{n}
$$

onde $n=3 ; A_{1}$ é um parâmetro empírico, que depende da temperatura do gelo, tamanho do cristal e orientação do gelo. Adotamos o valor $A_{1}=9.5129 * 10^{-25} \mathrm{~Pa}$; a tensão $\tau_{b}$ é calculada usando a equação (3.3) definida por

$$
\tau=\rho g \alpha Z
$$




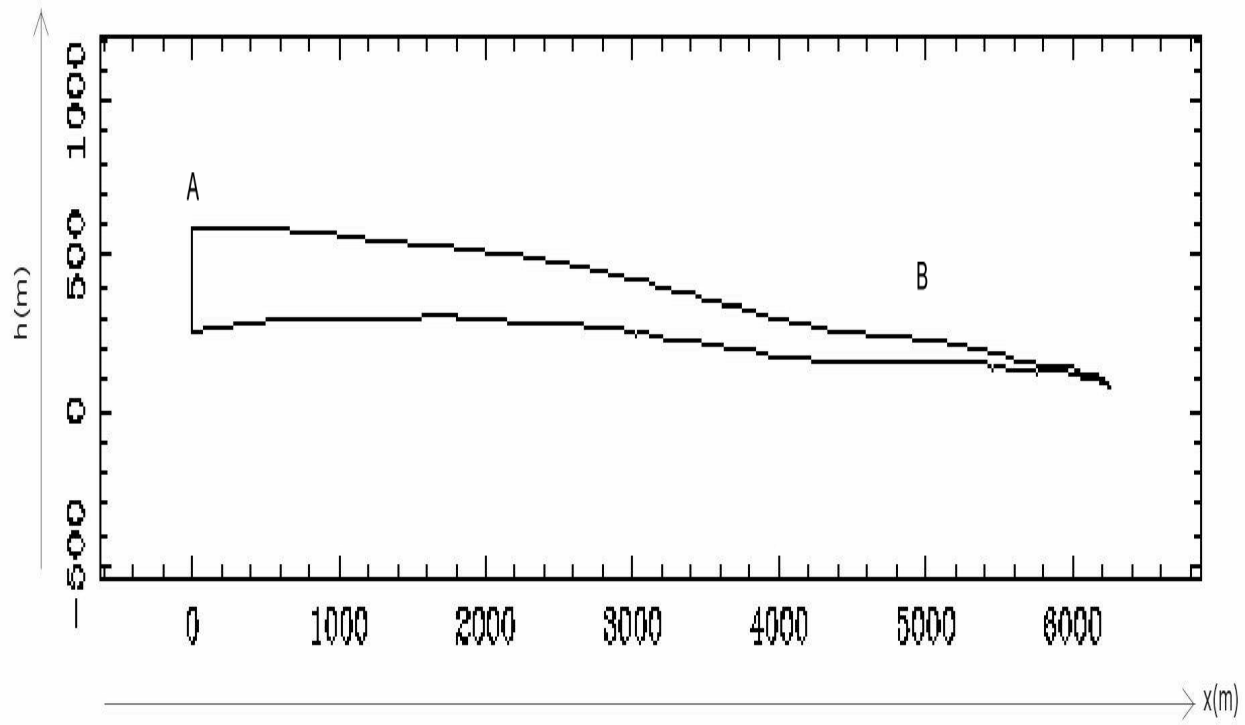

Figura 2: Perfil da Geleira Lange construído a partir do resultado da radioecossondagem ao longo do perfil A-B

onde $\rho$ é a média da massa específica do gelo, $g$ a aceleração gravitacional, $\alpha$ a declividade da superfície e Z a espessura do gelo. Para gelo de geleiras considera-se densidades entre 830 e $910 \mathrm{kgm}^{-3}([9])$. Neste trabalho adotou-se como densidade $900 \mathrm{kgm}^{-3}$.

A pressão sobre o leito basal, representada por $\tau$, é considerada como uma das forças envolvidas no movimento do gelo. Apesar da diferença de pressão longitudinal neste modelo seja considerada desprezível, porque consideramos apenas a deformação interna, é utilizada a Lei do Fluxo ([9]).

A equação da energia é parabólica para convecção fraca (baixas velocidades) permitindo o uso de diferenças centrais para os termos convectivos bem como para os difusivos ([4]). Por essa razão, o método de relaxações sucessivas (SUR- sucessive under relaxation) é empregado com coeficiente de relaxação de ordem 0.8 para obter variações de temperaturas (em torno de $1^{\circ} \mathrm{C}$ ) e velocidades coerentes com a situação física.

A equação da energia para um modelo bidimensional pode ser escrita em coordenadas cartesianas como,

$$
\frac{\partial T}{\partial t}+u \frac{\partial T}{\partial x}+v \frac{\partial T}{\partial y}=\frac{\kappa}{\rho C_{p}}\left(\frac{\partial^{2} T}{\partial x^{2}}+\frac{\partial^{2} T}{\partial y^{2}}\right)
$$

onde $T$ é a temperatura e $u$ e $v$ as componentes do vetor velocidade do fluxo nas 
direções $x$ e $y$; $t$ o tempo, $\kappa$ a condutividade térmica do gelo e $C_{p}$ o calor específico do gelo. As condições de contorno para a temperatura são do tipo Dirichlet.

As métricas oriundas da transformação de coordenadas indicam as relações entre o domínio físico e o computacional. Não é difícil mostrar que as relações entre elas são dadas por $([1],[3],[4])$

$$
\begin{gathered}
\xi_{x}=J y_{\eta}, \\
\xi_{y}=-J x_{\eta}, \\
\eta_{x}=-J y_{\xi}, \\
\eta_{y}=J x_{\xi},
\end{gathered}
$$

onde $J$ é o Jacobiano da transformação: uma relação entre as áreas em cada um dos espaços, ou seja,

$$
J=\frac{1}{x_{\xi} y_{\eta}-y_{\xi} x_{\eta}} .
$$

Com o objetivo de se obter uma solução numérica, condições de contorno apropriadas devem ser estabelecidas. Na superfície aberta uma condição de Dirichlet pode ser empregada porque alguns dados experimentais são disponíveis. Na superfície inferior e nas extremidades esquerdas e direitas do domínio os limites não são definidos completamente por experiências, sendo estimados. Com a equação da continuidade a mudança na espessura do gelo pode ser expressa.

A equação da continuidade é utilizada para expressar a mudança da espessura do gelo através da forma não linear da conservação do volume. Para o caso bidimensional, a equação torna-se

$$
\frac{\partial h}{\partial t}=b(x, t)-\frac{\partial q_{x}}{\partial x}
$$

onde $h$ é a espessura do gelo, $b$ é a taxa da acumulação e $q_{x}$ o fluxo do gelo, dado por

$$
q_{x}(x, t)=-2 A(n+2)^{-1}(\rho g)^{n}\left(\frac{\partial h_{s}}{\partial x}\right) h^{n+2}+h u_{b}(x, t)
$$

onde

$$
\alpha=\frac{\partial h_{s}}{\partial x}
$$

sendo $h_{s}$ a altitude e $A=6.810^{-15} \mathrm{~s}^{-1} \mathrm{~Pa}^{3}$. A integração temporal das equações (3.4) e (3.10) é feita utilizando o método de Runge-Kutta. 


\section{Resultados Numéricos}

Usando a equação da continuidade, verificamos a resposta da geleira Lange a alterações na atual taxa de acumulação de $0,6 \mathrm{~m}$ por ano em equivalente de água ([11]). Estes resultados foram obtidos para uma malha de $155 \times 6$ pontos e testes do refino da malha foram realizados visando esta escolha.

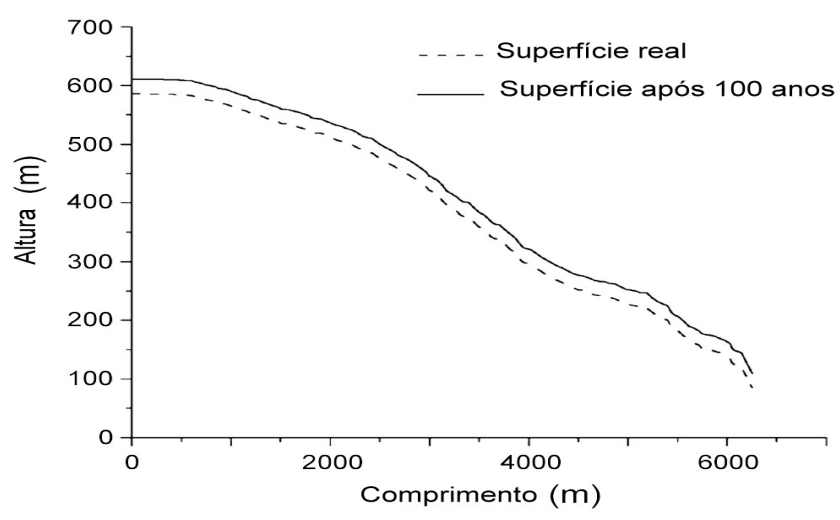

Figura 3: Variação da superfície da Geleira Lange para 100 anos com taxa de acumulação $50 \%$ maior do que a presente.

Numa simulação com o modelo durante 100 anos, para uma acumulação líquida avaliada em $20 \%$ a mais do que atual, não se modifica significativamente a superfície da geleira (Figura 4) ([3]). Um aumento líquido da taxa da acumulação de 50\%

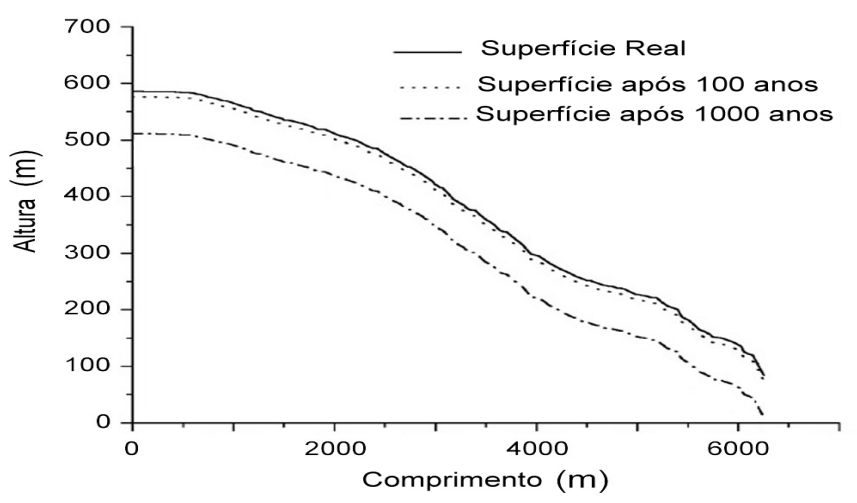

Figura 4: Variação da superfície da Geleira Lange para 100 e 1000 anos com uma taxa de acumulação líquida em torno de $20 \%$ menor do que a presente. 
resultaria em um aumento de aproximadamente $26 \mathrm{~m}$ no perfil da elevação (Figura 3) ([3]). Uma redução em $50 \%$ na taxa de acumulação diminuiria a elevação da geleira em $26 \mathrm{~m}$ depois de 100 anos.

\section{Conclusões Preliminares}

Este modelo pode fornecer informações qualitativas da velocidade ao longo da altura da geleira. Pequenas variações na taxa líquida da acumulação (isto é; menos de $20 \%$ do valor atual) não mudam significativamente a morfologia da superfície. O modelo quando simulado para 100 anos com uma redução de 50\% na taxa líquida da acumulação produz uma redução de $26 \mathrm{~m}$ da superfície antes de alcançar um novo estado de equilíbrio.

A deformação interna da geleira Lange não é muito sensível às variações na taxa líquida de acumulação, ao menos na escala temporal deste estudo (menos de 100 anos). O modelo mostra também que a geleira está perto do estado de equilíbrio, considerando as escalas de tempo envolvidas, mantendo-se desta forma até receber variações ambientais que afetem mais intensamente a geleira.

Embora os resultados sejam animadores, o modelo ainda necessita ser aprimorado, sendo necessário considerar outros fatores, como a corrente marítmica, que interferem na dinâmica das geleiras.

Abstract. During the last 40 years several glacier drainage basins of King George Island (Antarctica) have retreated, frequently more than one kilometer, losing about $7 \%$ of its original ice cover. Such ice loss occurred together with a mean atmospheric temperature increase of $1.1^{\circ} \mathrm{C}$. In this paper a simplified numerical model is used to simulate the flow of the outlet Lange Glacier (King George Island) to estimate its equilibrium state and morphological responses to snow accumulation rate changes. The model computes the velocity distribution along the main longitudinal axis $\mathrm{u}-$ sing the ice flow law to calculate the internal deformation, with no basal sliding, and responses to changes in the net accumulation rate. Results suggest a glacier near to the steady-state.

\section{Referências}

[1] D.A. Anderson, J.C. Tannehill e R.H. Pletcher, "Computational Fluid Mechanics and Heat Transfer", Mc Graw-Hill, New York, 1984.

[2] J. Arigony Neto, "Determinação e interpretação de características glaciológicas e geográficas com sistema de informações geográficas na Área Antártica Especialmente Gerenciada, Baía do Almirantado, Ilha Rei George, Antártica", Dissertação de Mestrado, Centro de Pesquisas em Sensoriamento Remoto e Meteorologia- UFRGS, Porto Alegre, RS, 2001.

[3] H.H.C. Barboza, "Simulação numérica bidimensional do fluxo da Geleira Lange via diferença finitas", Dissertação de Mestrado, UFRGS/PPGMAP, Porto Alegre, RS, 2002. 
[4] A.L. De Bortoli, "Introdução à dinâmica de fluidos computacional", Editora da UFRGS, Porto Alegre, 2000.

[5] F.A. Ferron, "Variações de isótopos estáveis na neve e no gelo da Ilha Rei George, Antártica", Curso de Pós Graduação em Geociências - UFRGS, Dissertação de Mestrado, Porto Alegre, R.S, 1999.

[6] Yu.Ya. Macharet, M.Yu. Moskalevsky, J.C. Simões e L. Ladouch, Study of King George Island Ice Cap, South Shetlands Island, Antarctica, Using RadioEcho Sounding and SPOT, ERS-1, SAR Sattelite Images, em "Proceedings of international seminar on the use and application of ERS in the Latin America", Viña Del Mar, Chile, vol. 49, pp.249-256, 1996.

[7] Yu.Ya. Macharet, M.Yu. Moskalevsky, J.C. Simões e L. Ladouch, Structure and Regime of the King George Island Ice Sheet, South Shetland Islands, Antarctica, as a Typical Glacier in the South Subpolar Region, Salzburger - Geographische Materialen, Austria, 28 (1998), 73-80.

[8] G. Ostrem e M. Brugman, "Glaciers mass-balance measurements, a manual for field and office work", National Hodrology Research Institute, Canadá, 1991.

[9] W.S.B. Paterson, "The Physics of Glaciers", Pergamon, Oxford, 1994.

[10] L.I. Sedov, "Dynamics of Snow and Ice Masses", Academic Press, USA, 1980.

[11] J.C. Simões, U.F. Bremer, F.E. Aquino e F.A. Ferron, Morphology and variations of glacial drainage basins in the King George Island Ice Field, Antarctica, Annals of Glaciology, 29 (1999), 220-224. 